This is the authors' accepted manuscript (accepted to be published in Journal of Voice, first published online 12 August 2019), (C) 2019 This manuscript version is made available under the CC-BY-NC-ND 4.0 license http://creativecommons.org/licenses/by-nc-nd/4.0/ DOI: 10.1016/j.jvoice.2019.07.019

\title{
Effects of humidification of the vocal tract and respiratory muscle training in women with voice symptoms - A pilot study
}

\author{
Kerttu Huttunen ${ }^{1,2,3,4}$ and Leena Rantala ${ }^{5}$ \\ ${ }^{1}$ Faculty of Humanities, Research Unit of Logopedics, University of Oulu, ${ }^{2}$ PEDEGO Research Unit, \\ University of Oulu, ${ }^{3} \mathrm{MRC}$ Oulu, and ${ }^{4}$ Department of Otorhinolaryngology, Head and Neck Surgery, \\ Oulu University Hospital, Oulu, Finland; \\ ${ }^{5}$ Degree Programme in Logopedics, University of Tampere, Tampere, Finland
}

\section{Corresponding author: Kerttu Huttunen}

Faculty of Humanities, Research Unit of Logopedics, University of Oulu

Address: P.O. Box 1000

FI-90014 Oulun yliopisto, Finland

email: kerttu.huttunen@oulu.fi

tel. +358504776610

Running head: Effects of humidification and respiratory training

\section{ABSTRACT}

Objective: The aim of this study was to determine the efficacy of a four-week breathing exercise intervention in participants with voice symptoms.

Methods: Six non-smoking women (mean age 49) experiencing voice symptoms used a novel device $\mathrm{WellO}_{2}{ }^{\mathrm{TM}}$ for respiratory exercises that provides counter pressure during both inspiration and expiration and warms and humidifies the breathing air. Speech samples were acoustically (Acoustic Voice Quality Index [AVQI]) and perceptually (grade, roughness, breathiness, asthenia and strain scale, [GRBAS]) analysed, and perceived voice symptoms and self-reported effort in breathing and phonation were obtained. Respiratory measurements included breathing frequency 
and pattern, peak expiratory flow (PEF), forced vital capacity (FVC) and forced expiratory volume in one minute (FEV1).

Results: The total scores of AVQI and some of its subcomponents (shimmer and harmonic-to-noise ratio), and the grade, roughness and strain of the GRBAS scale indicated significantly improved voice quality. However, neither the nature or frequency of the experienced voice symptoms nor the perceived phonatory effort changed as the function of intervention. According to the participants, their breathing was significantly less effortful after the intervention, although no significant changes were observed in the objective respiratory measurements with a spirometer. Conclusion: Training with the $\mathrm{WellO}_{2}$ device has the potential to improve voice quality. The combination of inspiratory and expiratory training and warmed, humidified air is a multifaceted entity influencing several parts in the physiology of voice production. The effects of using $\mathrm{WellO}_{2}$ need to be confirmed by further studies with a larger number of participants.

Keywords: Acoustic Voice Quality Index, functional voice disorder, dysphonia, pressure breathing, respiratory exercises, semi-occluded exercises 


\section{INTRODUCTION}

The function and health of both the respiratory and phonatory systems, in particular the hydration of the vocal fold mucosa, impact voice production. Problems in voice production can develop because of poorly coordinated breathing and phonation [1-3]. As a result of problems in coordination, there can be starting and ending phonation at lower than normal lung volume, shortness of breath, gasping for air during inhalation, forced expiration or decreased airflow during phonation $[1,4-8]$. If inspiratory air volume remains, for some reason, too low, a speaker may try to speak on residual air, using expiratory reserve volume. This increases muscle tension at the laryngeal level [2] and negatively affects voice quality [8]. In addition to its role as a contributing factor to dysphonia, abnormal respiratory behaviour can also be a sign of a compensatory mechanism. Sapienza, Stathopoulos and Brown (1997) [9] noticed that women with bilateral vocal nodules had higher glottal airflow and significantly greater than normal expenditure of air volume. They concluded that their participants were probably trying to compensate for the escape of air deriving from incomplete glottal closure. Vocal fold mucosa becoming dry, in turn, impairs vibration of vocal folds [10], and it is very common among persons with voice disorders to not to be well hydrated [8].

In many voice therapy methods, respiratory techniques are emphasized with which economic voice production and optimal glottal adduction can be achieved by adjusting subglottic air pressure and airflow [8]. In respiratory training, constant subglottic pressure is targeted to increase lung volumes for voice production and to control of smoother exhalation [5]. For instance, a patient with limited breath support can be asked to practice breathing for voice by saying as many numbers as possible on a normal expiration and stop before any strain emerges $[2,8]$. By using diaphragmatic (abdominal) breathing, downward contraction of the diaphragm and outward expansion of the abdomen permit greater airflow to the lungs and support voice production [5,8]. In Accent Method [11, see also 5], training based on paced rhythmic movements and accentuated abdominal breathing helps, according to Shiromoto (2003) [12], to improve controlling of cricothyroid, thyroarytenoid and abdominal muscles during phonation. By new motor speech breathing patterns learned, therapy aims to increased subglottic pressure [8]. Stronger subglottic pressure enhances an increased amplitude of vibration and a more stable adduction of vocal folds. Higher glottal efficiency can also be obtained by using semioccluded 
tubes. Using them a narrow constriction can be formed in the vocal tract and thereby raise mean supraglottic and intraglottic pressure [13-14]. Additionally, to prevent voice problems, the systemic hydration of the vocal folds by frequent water intake is commonly recommended $[2,8]$, especially for professional voice users [15].

The objective of this study was to determine if voice production and functioning of both the small and the large airways could be improved by a novel device $\mathrm{WellO}_{2}{ }^{\mathrm{TM}}$ (Figure 1 ) which, according to its manufacturer, ${ }^{1}$ simultaneously hydrates the respiratory tract and activates functioning of the respiration muscles. The $\mathrm{WellO}_{2}$ device is an innovation which combines counter pressure (resisted inhaling and exhaling exercises) and the simultaneous inhalation of warm steam.
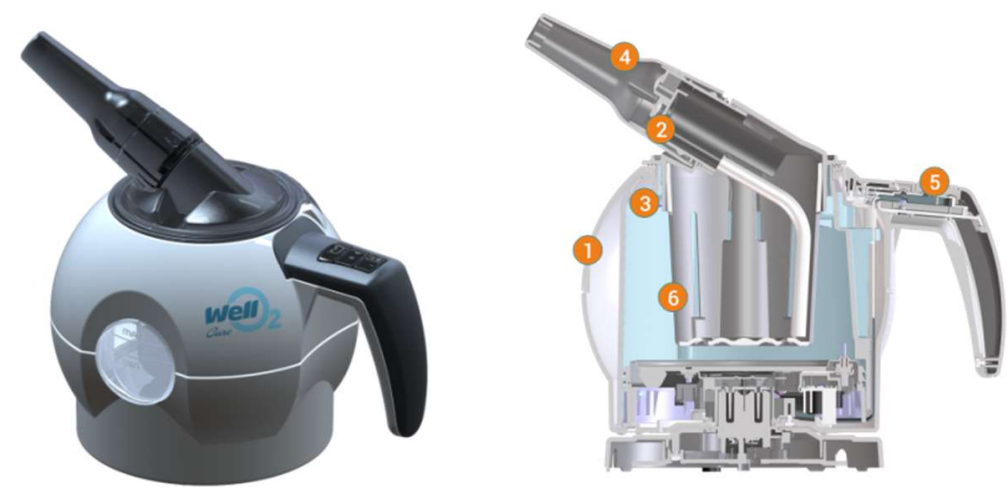

FIGURE 1.

\subsection{Humidification and voice production}

The condition of vocal fold mucosa is critical in voice production as, during vocalization, most of the vibratory function essential to voice quality occurs in the membranous portion of the vocal fold, the 1.1 to $2.5 \mathrm{~mm}$ thick mucosa that covers the thyroarytenoid muscle $[8,16]$. In Nordic parts of the world, particularly cold and dry air loads the vocal organs because the ability of air to hold moisture is quickly impaired with declining temperature. Cold air and abrupt temperature changes also increase the risk of vocal tract mucosa inflammations (common cold). Additionally, strong and prolonged voice production causes tissues to dry as capillaries contract when the vocal folds collide with each other [17]. Because of the high frequency of vocal fold vibration, women's relatively thin vocal folds become dry very easily, and this dry tissue is especially vulnerable to

\footnotetext{
${ }^{1}$ http://wello2.com/product/ and http://wello2.com/faq/
} 
damage [18]. Titze and Hunter (2015) [19] point out, however, that although women, compared to men, have higher vocal fold vibration frequency, they have lower vibrational amplitude and often a smaller contact quotient. Energy dose related to vocal fold collision is highly dependent on tissue viscosity, and more research is needed to find out the relations between viscosity, amplitude, and vocal-fold thickness in vibration and collision.

Dry indoor air also increases the occurrence of voice symptoms [20] and makes a speaker feel that one needs to make a special effort to vocalize [21-22]. Voice symptoms are caused by the stiffening and increased viscosity of the surface of the mucosa when it becomes dry [23]. Greater than normal subglottic air pressure is needed to get the dry mucosa to begin to vibrate [21-22] which means increased effort needed in voice production. In the long run, increased effort to produce voice may lead to vocal fatigue, laryngeal pathology, or both [8]. In dry indoor air (relative humidity in air 15-25\%), oral breathing that lasts only for 15 minutes may increase phonation threshold pressure in speakers with voice symptoms [24]. Mucosal wave amplitude has also been detected to decrease in dry air [25]. In turn, hydration treatment tends to reduce phonation threshold pressure, though the effect is greater in vocally healthy speakers than those with pathological voice [26]. There is no clear evidence that one type of osmotic nebulizer (hypertonic, isotonic saline or sterile water) would be more effective than another in affecting phonation threshold pressure (PTP) [22].

Both surface and systemic hydration of vocal folds can be used as a voice ergonomic measure, as well as a treatment in voice therapy. The laryngeal surface is hydrated by using steam inhalation or spending time in a humidified environment, whereas in systemic hydration, liquids, mostly water, are drunk to hydrate the whole body. The recommendation to drink plenty of water is an integral part of vocal hygiene measures [27]. The significance of hydration for voice quality has been investigated in some studies with the means of hydrating or dehydrating vocal organs. Overall, however, there has been a paucity of research on the humidification of voice organs, and despite the good quality of the existing studies, robust evidence for the effectiveness of hydration treatments has been lacking until recently [28], and the results have also been somewhat contradictory $[10,29]$. Studies exploring the effects of laryngeal humidification on voice quality and its acoustically measured parameters have been particularly sparse. The few interventions 
reported have most often been short-term; hardly any reports have been published on long-term interventions on the hydration of the vocal tract or vocal folds.

Based on our literature search, the two longest humidification interventions lasted for five days [30] and for one month [31], respectively. In the former study, participants with vocal nodules or vocal polyps first drank water and breathed moist air (relative humidity of 90-100\%) for two hours a day for five days, which was followed by five days of placebo intervention [30]. The hydration treatment was found to be more effective than the placebo treatment. In the latter study comprising a one-month long hydration intervention [31], 27 teachers breathed nebulized water containing saline solution for five minutes once or twice during their working day. This hydration took place after dehydration was induced by a 10-minute period of breathing through the mouth. The intensity of connected speech of the participants was found to decrease, and fundamental frequency of sustained vowel production to increase. An increase in fundamental frequency has been considered to reflect vocal folds vibrating more quickly when well lubricated [29]. However, no significant changes were found either in further acoustic parameters (jitter, shimmer) or in the auditory-perceptual evaluation of the overall disorder severity degree parameter of Consensus Auditory Perceptual Evaluation - Voice (CAPE-V) [31].

\subsection{Breathing exercises and voice production}

Volume of air in the lungs, subglottic pressure and airflow rate through the glottis influence the efficiency of vocal production [32]. The amount of inhaled air needs to be enough for speaking without any special effort used for voice production. The longer the utterance produced within a breathing cycle, the more a speaker needs air, and the more fluently he or she needs to adjust the functioning of the breathing muscles. Activation of expiratory muscles is needed to increase loudness of voice; in loud vocalization, tidal air may increase from the ordinary half-litre up to four or even five litres.

Breathing manner has also been found to influence laryngeal functions [32]. During inhalation, the diaphragm contracts and moves downwards and thereby increases the volume of the thoracic cavity. With the help of this process, inhaled air reaches the lower parts of the lungs. The strong downwards movement of the diaphragm causes the traceal pull; the larynx is pulled downwards, 
which increases the volume of the laryngeal area and improves the vibration possibilities of the vocal folds [33-34]. However, in the case of a fairly small lung volume, the quality of voice easily turns hyperfunctional [35].

Individuals with voice disorders have been found to use tidal air in a manner that is different from those with healthy voices. Compared to normal volumes, speakers with organic voice disorders have low lung volume both before [4] and after [4,36] producing an utterance. On the other hand, it has also been found that patients with vocal nodules inhale more air than is normally done before speaking $[9,37]$. Persons with a functional voice disorder have also been described to have a deviant breathing habit: they start and end speaking with lower than normal air volume [6].

Because respiratory musculature represents skeletal muscles and is voluntarily controlled by the somatic nervous system [15], its strength can be increased by training [38]. The study of Tsai and colleagues [39] showed that it is possible to decrease vocal symptoms by using breathing exercises only. Participants $(N=29)$ working in hospital and using their voice for more than four hours per day performed 25 expiratory exercises three days per week for five weeks. After the intervention, the participants' self-reported voice symptoms were found to be reduced. Additionally, their voice production was improved, which was shown by the increased maximal expiratory pressure and increased phonation time both in the voiceless fricative [s] and in the voiced fricative [z]. On the other hand, no change in voice symptoms was found in teachers $(N=20)$ who trained their expiratory muscles with an expiratory pressure threshold trainer by exhaling vigorously 25 times per day five days a week over six weeks [40].

The effects of combined breathing and voice exercises were explored in the intervention study of Wingate et al. [41]. Two groups of voice patients $(N=9+9)$, representing professional voice users, participated in the intervention. First, one group had expiratory muscle strength training with an expiratory pressure threshold trainer for five weeks, and the breathing intervention was then followed by six sessions of traditional voice therapy. The other group went through the same treatment in reverse order. The combined treatment was found to be successful in remediating voice problems; the expiratory muscle strength training improved maximal expiratory pressure. Treatment order had no significant effect on the positive results obtained. According to Wingate 
and colleagues [41], training of expiratory muscles supports voice production and improves voice quality in situations where a speaker needs to use his or her voice for prolonged periods of time.

\subsection{Aim of the study}

This study aimed to find out whether simultaneous exercising of respiratory muscles and warming and humidifying of vocal tract mucosa affect voice production and breathing in adults with voice symptoms. Generally, pressure breathing devices provide counter pressure only for inhalation or exhalation, but with the $\mathrm{WellO}_{2}$ device (http://wello2.com/) used in the present study, pressure can be used in training both inspiratory and expiratory muscles. Additionally, the $\mathrm{Well}_{2}$ device warms and moistens breathing air and, in that way, is expected to open up the airway and remove phlegm.

\section{MATERIAL AND METHODS}

The Regional Ethical Board of Tampere University Hospital, Finland approved the study protocol. Additionally, Valvira, the National Supervisory Authority for Welfare and Health, accepted the notice submitted to them as a health and safety procedure. Participants gave their written informed consent to the study protocol. Originally, $\mathrm{WellO}_{2}$, the novel device used, was developed to ease breathing of patients with asthma, but it is also used by others such as athletes, and singers and other professional voice users.

\subsection{Participants}

Six women aged $28-58$ years with a mean age of 49 years (SD 11.1) who complained of voice symptoms participated in the study. They had contacted researchers after becoming aware of the research notice about the study and the contact request that had been sent to local speech and language therapists, as well as to venues such as schools. The recruited participants worked in kindergartens, schools and hospitals and needed to use their voice frequently during their working day. Three of the participants were teachers. All participants were non-smokers, and five drank coffee, in most cases one to three cups daily. 
Self-reported vocal symptoms were used as the inclusion criterion. Symptoms of all the participants had lasted for at least more than month, often for years. Participants were excluded if they had acute infection symptoms of the upper respiratory tract or symptoms of respiratory allergy during the initial or final measurements. Persons with psychogenic voice problems, known organic vocal fold pathologies or neuromotor diseases and conditions related to cerebral blood circulation were also excluded from the study. In addition, contraindications to use the $\mathrm{WellO}_{2}$ device listed in its instructions ${ }^{2}$ automatically resulted in exclusion from the study. These contraindications included unstable cardiovascular status, recent myocardial infarction or pulmonary embolus, pneumothorax, pulmonary emphysema, known thoracic, abdominal or cerebral aneurysms, epilepsy, recurrent episodes of nose bleeding, recent eye, thorax or abdomen surgeries and pregnancy. These conditions could cause a health risk because even a relatively high counter pressure may be present in inhalation and exhalation when the $\mathrm{WellO}_{2}$ device is used.

Two participants had been examined by a phoniatrician, and they were found to have incomplete vocal fold closure. Five participants had been given an asthma diagnosis, and five (one of them without an official asthma diagnosis) used asthma medication either regularly or as needed. Two reported cough or voice symptoms caused by asthma medication. Additionally, one suffered from respiratory allergy, and three had other allergic symptoms. Three participants had received voice therapy previously, mostly years ago, but none engaged in on-going therapy during the intervention with the $\mathrm{WellO}_{2}$ device.

Based on their reports, all the participants had voice ergonomic risk factors in their workplaces. Workplaces were often noisy (noise occurring weekly; 4 participants), and the indoor air was dry (4 participants), stuffy (3 participants) or dusty (3 participants). Three participants were exposed to draughts in their workplaces.

\subsection{Intervention: Training with the $\mathrm{WellO}_{2}$ device}

The intervention was carried out during the winter season when breathing air is dry because of the cold weather outside and the heating required indoors. The participants were requested to train with the $\mathrm{WellO}_{2}$ device ten minutes per day for four weeks. When the $\mathrm{WellO}_{2}$ device was given to

\footnotetext{
${ }^{2}$ http://wello2.com/wp-content/uploads/wello2_care_-_instructions_for_use_ver_1-02.pdf
} 
the participants, they were individually instructed on how to use it. Additionally, the participants had the $\mathrm{WellO}_{2}$ manual available both in paper form and as an on-line version at the manufacturer's Internet site. The Internet site (in Finnish: http://wello2.com/fi/tuki/ and in some other languages: http://wello2.com/support/) also contains a video demonstration on the use of the device. Furthermore, the participants had the ability to contact the researchers during the intervention whenever they had questions about the training or the device.

The participants themselves could choose the temperature of inhalation steam and the strength of the counter pressure (both on a scale from zero to three). We decided to allow free choice of temperature and counter pressure settings. Firstly, we expected the participants of our study to have individual sensitivity to and liking for steam temperature of the $\mathrm{WellO}_{2}$ device. Steam perceived to be too hot may be a stressor which would not serve the aims of remediating or relieving voice problems. Secondly, we did not know in advance how the diagnosed asthma of five participants would affect their ability to breath using the counter pressure. The fixed temperature settings available for steam with the $\mathrm{WellO}_{2}$ device were 48,58 and $63^{\circ} \mathrm{C}\left(118,136\right.$ and $145^{\circ} \mathrm{F}$, respectively). The minimum setting (zero) for the counter pressure was $20 \mathrm{cmH}_{2} \mathrm{O}$, matching a normal blow when the lungs deflate from air during an approximately 15-second-long exhalation. The other available counter pressure settings were 30,50 and $100 \mathrm{cmH}_{2} \mathrm{O}$. With the $\mathrm{WellO}_{2}$ device, counter pressure increases with stronger inhalation or expiration. During inhalation, the counter pressure is about one-third of the counter pressure that prevails during exhalation.

A WellO $\mathrm{O}_{2}$ accessory, a $40-\mathrm{cm}$-long flexible extension hose having an inside diameter of $1 \mathrm{~cm}$, was requested to be used in training. Use of the hose enhances a good, straight posture for breathing exercises, which, in turn, allows space for expansion of the rib cage and the diaphragm. The hose also cools down the breathed warm air and increases counter pressure because, by using the hose, the distance between the device and the user increases.

The participants were asked to complete a daily recording of the time used for training and the temperature and counter pressure settings most frequently used. They were also asked to record any special sensations, possible infections, changes in medication etc. during the intervention. After the intervention, the participants were also interviewed about their views and experiences in using the $\mathrm{WellO}_{2}$ device. 


\subsection{Data collection and analysis}

Data were collected before the four-week intervention and right after it; thus, participants served as their own controls. Data were collected using the following methods.

\subsubsection{Voice symptoms and voice measurements}

The vocal symptoms were surveyed with a questionnaire. The symptoms queried about were as follows: 1) voice tires easily, 2) voice becomes hoarse or low, 3) voice breaks, 4) aphonia lasting at least a couple of minutes during speaking, 5) difficulty in being heard, 6) throat clearing and 7) sore throat or globus in the throat. These symptoms have been found to reliably distinguish voice disorders from healthy voice [42], and they have been used in several studies in which vocal health has been researched [42-48]. Symptoms occurring weekly or almost weekly or daily or almost daily were inquired.

Voice samples. Two different voice samples were recorded: three five-second-long sustained vowels [a] and reading of a standard text ('The North Wind and the Sun'). The sustained vowels were asked to be produced with a habitual pitch and loudness of speaking voice. Additionally, samples from spontaneous speech were collected when the participants described their voice and vocal health (pre- and post-intervention) and, in addition to that, reported on their experiences with the $\mathrm{WellO}_{2}$ device (post-intervention). More specifically, spontaneous speech samples consisted of responses to relevant questions: 'How is your voice feeling today?', 'What do you think about your voice?' and, after the intervention phase: 'How did the training with the $\mathrm{WellO}_{2}$ device go?'.

The voice samples were recorded with an external Focusrite iTrack Solo (Focusrite Audio Engineering Plc, London, Great Britain) sound card connected to a laptop computer and an AKG C544L headset microphone (AKG Acoustics, Vienna, Austria) at an angle of $45^{\circ}$ and a distance of 4 $\mathrm{cm}$ from the corner of the mouth. The material was recorded and saved in wave format with $44,100 \mathrm{~Hz}$ sample rate and 16-bit depth. The recordings were made in office or class facilities either at the university or at the participants' workplaces and performed the same time of the 
day in each subject because vocal symptoms are known to increase towards the end of the working day [49-50].

Perceived phonatory effort (PPE). The participants assessed their PPE immediately after the voice tasks (see Voice samples) by using a 10-cm-long visual analogue scale (VAS) where (at the extreme ends of the scale) 0 represented 'no effort' and 100 represented 'extreme effort' with higher scores indicating more effort needed for voice production (see, e.g. [22]). In the post-intervention measurements, new, blank forms with VAS scores were used to prevent the participants from seeing their earlier ratings.

Acoustic analysis. Quality of the voice was assessed with Acoustic Voice Quality Index (AVQI) version 03.01. For the AVQI analyses, a sequence of three seconds was manually extracted from the middle of the five-second-long sustained vowel [a] together with the first 12 words (this 12word text sequence consisted of 31 syllables) read from the beginning of the standard text. AVQI total score is a multiparameter method developed to measure the general quality of voice, also known as grade (G), from sustained vowel production and continuous speech [51], and it has been found to be reliable, valid and capable of distinguishing dysphonic and normophonic voices [5253]. To date, its versions have already been validated in many European languages, including Finnish [54]. The index, expressed on a scale from 1 to 10, comprises a weighted combination of six acoustic parameters [55]. Two of the parameters measure noisiness (smoothed cepstral prominence and harmonics-to-noise ratio $[H N R])$, two measure time or regularity of amplitude (local shimmer; in percent and in decibels), and two measure the voice quality in a hyperhypofunctional continuum (general slope of the spectrum and tilt of the regression line through the spectrum). AVQI script version 03.01 [56], developed for the Praat [57] acoustic analysis programme, was used in the analysis. An AVQI 03.01 total score exceeding 1.83 indicates voice disorder in the Finnish speaking population [Kankare, manuscript].

Auditory-perceptual assessment. Three voice samples were used in the auditory-perceptual assessment. Two were the same as the ones used in the acoustic analysis (the middle part of sustained vowel and a 12-word sequence from the text reading), and the third one was a ten-word sequence from spontaneous speech. For the spontaneous samples, care was taken that the contents of the segmented speech sample did not reveal whether the sample was recorded before 
or after the intervention. The three voice samples deriving from pre- and post-intervention were each collected to form one block. The order of the two blocks obtained before and after the intervention was randomized by using a random number generator, and the blocks were placed one after the other with a two-second inter-stimulus interval. Thus, the judges could listen to the samples as many times as they wished to and compare samples from the two time points with each other.

Five speech and language therapists with a long experience in assessing and treating voice disorders assessed the randomized and blinded speech samples. For practicing, the judges were first asked to assess voice samples of a female teacher who was not one of the participants, but whose voice symptoms matched those of the participants of the present study. Voice quality in the samples at each data collection point was first assessed with the grade, roughness, breathiness, asthenia and strain (GRBAS) scale [58] used to measure dysphonia according to four severity classes (normal, slight, moderate and severe). The same components were also evaluated using a 10-cm-long VAS with 'not deviant' and 'extremely deviant' as descriptions of the extreme ends of the line. The use of additional analyses using the VAS was opted for because functional voice disorders may not show very high symptom severity on the four-point GRBAS scale, and a possible change in symptom severity would therefore not necessarily be tapped with the GRBAS scale representing a discrete parameter. Additionally, the grade component of the GRBAS scale used in the traditional manner has been found to strongly correlate with the CAPE-V method utilizing VAS [59].

\subsubsection{Breathing measurements}

Perceived pulmonary effort. The participants assessed their perceived breathing effort immediately after they had given voice tasks (see Voice samples) by using a VAS in a similar way they assessed their phonatory effort. They did it by using a 10-cm-long VAS (see, e.g. [22]) with 'no effort' and 'extreme effort' at the extreme ends of the line. Higher scores indicated more effort needed for breathing. Again, in the post-intervention measurements, new, blank forms with VAS were used.

Pulmonary function testing. Expiratory and inspiratory flow measures reflecting respiratory muscle strength and respiratory force were measured with forced vital capacity (FVC) and forced 
expiratory volume in one second (FEV1) by using a hand-held Contec SP10 digital spirometer (Contec Medical, Qinhuangdao, China). Calibration of the spirometer was checked with a threelitre calibration pump, and the measured volume was found to be acceptable, that is, within $1 \%$ of the target calibration pump volume. Additionally, Joshi and Watts [60] determined that vital capacity measurements done with this hand-held SP10 are highly reliable when compared with a pneumotachograph-based instrument (Pentax Medical Phonatory Aerodynamic System [PAS]: Model 6600, Tokyo, Japan).

Peak expiratory flow (PEF). PEF reflects the spaciousness of the airway, strength of the breathing muscles and elasticity of the lungs. It was measured in this study with MiniWright $60-800 \mathrm{I} / \mathrm{min}$ meter for adults (Clement Clarke International, Harlow, Great Britain) which is compliant with EN 23747 Standard.

Spirometry and PEF measurements were performed using the guidelines of the American Thoracic Society/European Respiratory Society [61] and the Finnish Society of Clinical Physiology and Finnish Respiratory Society [62]. Disposable cardboard mouthpieces were used to avoid crossinfections among the participants.

\subsection{Statistical analyses}

The IBM SPSS Statistics 24 for Windows (IBM, Armonk, NY, USA) was used in the analyses. The non-parametric Wilcoxon signed-rank test was used as the statistical test to explore the possible changes as a function of the $\mathrm{WellO}_{2}$ intervention. Effect size calculations were based on Cohen's $d$ (applied using the Wilcoxon signed-rank test $Z$ scores). Effect sizes exceeding 0.5 were considered to represent large to very large magnitudes [63].

Regarding the auditory-perceptual assessment of the speech samples, Cronbach's alpha was calculated for reliability of the judgments of the grade component of GRBAS, and intraclass correlation coefficient (ICC) estimates with their 95\% confidence intervals based on mean-rating, consistency and a 2-way mixed-effects model were used in analysing the reliability of using the GRBAS components on the VAS scale. Koo and Li [64] suggest that inter-rater agreement is poor if ICC values are less than 0.5 , moderate with ICC estimates between 0.5 and 75 and good with ICC 
estimates between 0.75 and 0.9. Spearman's rho was used in finding out the correspondence of grade component of the ordinal-scale GRBAS and $G$ assessed using the VAS scale. An alpha value of 0.05 was adopted as the value for statistical significance.

\section{RESULTS}

According to their self-reports, the participants used the $\mathrm{WellO}_{2}$ device during the four-week intervention for, on average, 256 min (SD 33.57), that is, a bit over four hours. At minimum, the duration using the device was $225 \mathrm{~min}$, and at maximum, $315 \mathrm{~min}$. Mean duration of the daily exercising was $10 \mathrm{~min}$ (SD 0.52). The mean number of days the $\mathrm{WellO}_{2}$ device was used was 26 (SD 3.34), and the most typically selected temperature was 2 (scale $0-3$ ) and counter pressure 2 (scale $0-3)$.

\subsection{Voice symptoms and PPE}

According to their self-reports, our participants had mostly mild voice disorders; three of them suffered from three vocal symptoms most weeks or more frequently, and one had one vocal symptom that emerged daily or most days. The remaining two had symptoms occurring monthly or less often. The most typical symptom of the participants was that their voice became hoarse. Other frequently occurring (at least weekly or most weeks) symptoms were the voice becoming tired, and there also was a frequent need to clear the throat. The participants experienced these symptoms both before and during the intervention period, and between these periods, no significant difference was found in the nature and frequency of self-perceived vocal symptoms.

PPE was reported using a VAS scale right after recording of the voice samples. No significant difference was found between the assessment preceding (mean 59, SD 8.91) and following (mean 43, SD 25.67) the intervention, although four out of six participants did report voice production to be less effortful after the intervention with the mean improvement being 17 (SD 26.46). 


\subsection{Voice quality}

\subsubsection{AVQI total score and its parameters}

The AVQI 03.01 total scores were over the cut-off level in all the participants before the $\mathrm{WellO}_{2}$ intervention (mean 3.30, SD 1.36), indicating that they had a voice disorder (see Figure 2 and Table 1). Dysphonia severity was reduced after the intervention in five participants with two participants reaching the normal value. Improvement in the AVQI total score was significant with a large treatment effect $(r=-0.576)$. HNR, shimmer local (\%) and shimmer local (dB) also improved significantly with $p<0.05$ in both cases. Effect size for HNR change was 0.636 , for shimmer local (\%) change -0.636 , and for shimmer local $(d B)$ change -0.638 .

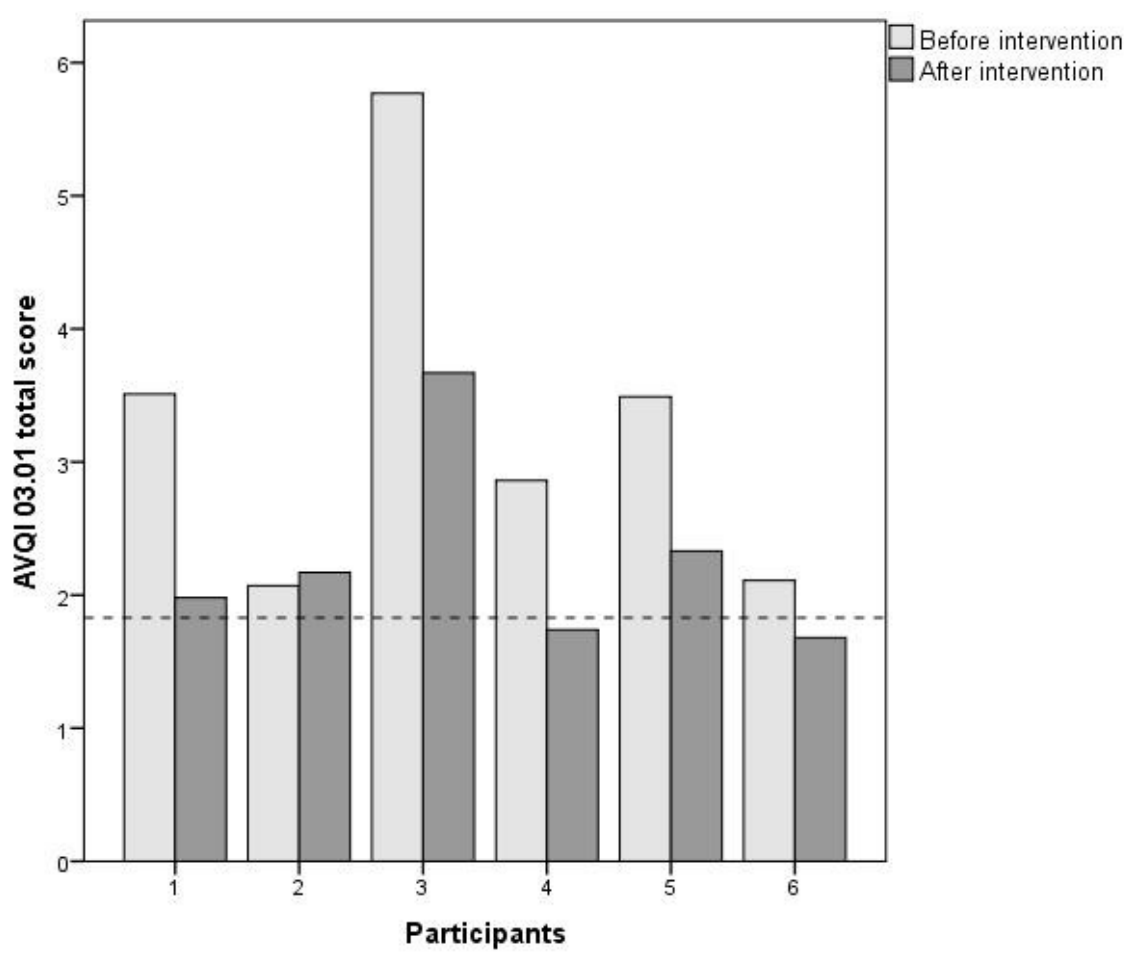

FIGURE 2. 
TABLE 1. Mean and SD of the AVQI 03.01 Total Score and the Six Parameters Constituting It before the Four-Week Respiratory Training and after It

\begin{tabular}{|c|c|c|c|c|c|c|c|c|}
\hline & \multicolumn{3}{|c|}{ Before } & \multicolumn{3}{|c|}{ After } & \multirow[t]{2}{*}{$Z$} & \multirow[t]{2}{*}{$P$} \\
\hline & Mean & $S D$ & Min-max & Mean & $S D$ & Min-max & & \\
\hline $\begin{array}{l}\text { AVQI total } \\
\text { score }\end{array}$ & 3.30 & 1.36 & $2.07-5.77$ & 2.26 & 0.73 & $1.68-3.67$ & -1.992 & 0.046 \\
\hline CPPS & 11.43 & 1.85 & $8.19-12.84$ & 12.27 & 1.18 & $10.36-13.47$ & 1.363 & ns \\
\hline HNR & 18.59 & 1.67 & $16.11-20.77$ & 20.35 & 1.58 & $18.51-22.68$ & 2.201 & 0.028 \\
\hline $\begin{array}{l}\text { Shimmer } \\
\text { local (\%) }\end{array}$ & 6.83 & 2.74 & $3.86-11.05$ & 4.28 & 0.65 & $3.62-5.31$ & -2.201 & 0.028 \\
\hline $\begin{array}{l}\text { Shimmer } \\
\text { local (dB) }\end{array}$ & 0.69 & 0.29 & $0.38-1.12$ & 0.44 & 0.05 & $0.37-0.52$ & -2.207 & 0.027 \\
\hline $\begin{array}{l}\text { LTAS } \\
\text { slope }\end{array}$ & -25.56 & 2.54 & $\begin{array}{l}-28.76- \\
-21.02\end{array}$ & -25.79 & 3.28 & $\begin{array}{l}-30.16- \\
-20.88\end{array}$ & 0.314 & ns \\
\hline LTAS tilt & -11.67 & 0.55 & $\begin{array}{l}-12.21- \\
-10.62\end{array}$ & -11.53 & 0.52 & $\begin{array}{l}-12.14- \\
-10.62 \\
\end{array}$ & 1.753 & ns \\
\hline
\end{tabular}

CPPS $=$ Smoothed cepstral peak prominence, $\mathrm{HNR}=$ Harmonics-to-noise ratio, LTAS slope $=$ Slope of longterm average of spectrum, LTAS tilt = Tilt of trend line through long term average of spectrum.

\subsubsection{Perceptual assessment}

Cronbach's alpha (internal consistency) for the perceptually assessed grade component of the ordinal-scale GRBAS was 0.825 before and 0.455 after the intervention. Reliability of the listener judgments in all components of the GRBAS scale on VAS was analysed with ICC. ICC estimates ranged from 0.67 for $\mathrm{S}$ with the $95 \% \mathrm{Cl}[-0.099,0.947]$ to 0.86 for $\mathrm{B}$ with $95 \% \mathrm{Cl}[0.539,0.978]$ before the intervention, and from 0.34 for $\mathrm{A}$ with $95 \% \mathrm{Cl}[-1.989,0.895]$ to 0.72 for $\mathrm{B}$ with $95 \% \mathrm{Cl}$ $[0.071,0.995]$ after the $\mathrm{WellO}_{2}$ intervention.

Before intervention, participants' scores on the GRBAS scale, based on the mean of five listeners' evaluation, ranged from G1R1B0A0S0 to G2R1B2A1S2, with the mean values of 1.23 (SD 0.817 ) for grade, the most affected component. After the intervention, mean of the grade component among the six participants was $1.03(0.669)$ indicating improvement, although the change was not significant.

The mean VAS value of the perceptual assessment was 37 (SD 22.89) before and 30 (SD 19.70) after the intervention with the improvement being significant (see Table 2). The values for roughness and strain were also significantly lower after the training period compared to the situation prior to training. Effect sizes for the changes were large: for grade -0.636 , for roughness - 
0.585, and for strain -0.576. Grade component of the ordinal-scale GRBAS and on VAS correlated strongly before $(r=0.986, \mathrm{p}<0.001)$ and after $(r=0.853, \mathrm{p}<0.05)$ the intervention.

TABLE 2. The Values for the GRBAS Components on VAS before and after the Four-Week Intervention. The Higher the Value, the Poorer the Voice Quality

\begin{tabular}{lcccccccc}
\hline & \multicolumn{3}{c}{ Before } & \multicolumn{3}{c}{ After } & $\boldsymbol{Z}$ & $\boldsymbol{P}$ \\
\hline & Mean & SD & Min-max & Mean & SD & Min-max & & \\
Grade (G) & 36.9 & 22.89 & $11-84$ & 30.2 & 19.70 & $4-83$ & -2.201 & 0.028 \\
Roughness (R) & 29.3 & 22.40 & $0-82$ & 22.1 & 22.79 & $0-81$ & -2.023 & 0.043 \\
Breathiness (B) & 31.7 & 22.72 & $0-82$ & 27.4 & 20.18 & $0-81$ & -1.153 & ns \\
Asthenicity (A) & 18.7 & 22.00 & $0-82$ & 14.7 & 19.40 & $0-80$ & -1.472 & ns \\
Strain (S) & 34.3 & 21.35 & $1-90$ & 23.5 & 21.69 & $0-85$ & -1.992 & 0.046 \\
\hline
\end{tabular}

\subsection{Breathing measurements}

As observed before the intervention, breathing frequency of the participants was, on average, 12.7 breaths/min (SD 1.63), which is considered normal, with ideal breathing frequency of adults being 10 to $14 \mathrm{cycles} / \mathrm{min}$ [65]. Breathing frequency did not significantly change after the intervention (mean 10.8 cycles/min) compared to pre-intervention measurement results. The initial PEF values (mean 457 litres/min, SD 52.79), individually proportioned to age and height, were also within the normal range $[62,66]$ even though five of the participants had asthma. No significant difference was found between the time point before and after the $\mathrm{WellO}_{2}$ intervention in the measurements of FVC or FEV1. Both pre- and post-intervention mean values were below the reference values (FVC mean 3.65, SD 0.68; FVC1 mean 2.91, SD 0.61) of Finnish healthy nonsmoking female adults $(N=613)[66]$.

All six participants perceived their breathing to be less effortful at post-intervention when asked immediately after the voice tasks $(Z=-2.201, p=0.028)$. According to VAS values, the effort decreased by 24 scores (SD 16.70) from the pre-intervention (mean 45, SD 27.36) to postintervention (mean 21, SD 16.83). 


\section{DISCUSSION}

Our study showed that a four-week training that activated respiratory muscles and hydrated the surface of vocal folds was able to improve voice quality, even though no direct vocal exercises were included in the exercise programme. Objective and perceptual assessment variables indicated improvement in voice. AVQI total score and shimmer were lower and HNR was higher (with moderate effect sizes) after the intervention than before it. The listeners, who were speech and language therapists, experienced in perceptually assessing voice disorders, heard recuperation in the overall voice quality, roughness and strain assessed using VAS. The mean value of the overall voice quality decreased by 6.7 scores on the VAS scale and was 30 after the intervention. If the cut-off point, 34 , for the disordered voice is applied, as used by Uloza et al. [67], our participants' voices were healthy on the group level after the intervention with a large effect size. The participants also reported their breathing to be less effortful after the training. However, the participants' voice symptoms and perceived phonatory effort did not change during the intervention. The impact of the small number of participants and the resulting large standard deviation on these results need to be considered.

In our intervention, there were two factors that influenced the voice production system: laryngeal hydration with warm steam and expiratory muscle strength training. As far as we know, there are no previous studies combining these two interventions. Therefore, we now compare the effects of the two methods with earlier results separately. A comprehensive comparison of our results will also remain limited because the variables applied by the two interventions that we combined have not been identical in research literature: voice related parameters have mostly been measured in hydration interventions (see [29]), whereas expiratory flow or pressure have been measured when training breathing (see, e.g. [68]). Hence, a reader needs to keep in mind that both hydration and expiratory training have affected our results at the same time, although we discuss the two methods separately. 


\subsection{The effects of hydration}

Our results are mainly in line with the findings of the studies that have assessed the effects of hydration on the vocal tract. In particular, shimmer seems to be a parameter that reacts to the hydration level: it decreases when vocal folds are hydrated [69-70] as in our study and increases when they are desiccated $[69,71]$. The effect has been found both in surface [69] and systemic [70] hydration/dehydration interventions. Decreased shimmer values indicate more even vocal fold vibration and healthier mucosa of the vocal folds.

In addition, we found that an increased HNR also reflected improved voice quality. This has also been verified previously [69], although not always (systemic hydration: see [30,72], surface hydration: see $[31,71])$. Increased HNR is known to correspond with the perceptual assessment of decreased roughness [73], the change of which the listeners also observed in the present study.

The other acoustic variables - CCPS and slope and tilt of LTAS - did not change during our intervention. This outcome was not what we expected because these parameters also express noisiness (aperiodicity) in a sound just as HNR does. The discrepancy may originate from the fact that the parameters measure noisiness in different ways by using different algorithms. Further, these acoustic parameters have also been shown to correlate with different perceptual voice qualities: CCPS manifests breathiness [74], whereas LTAS variables reflect hypo-hyperfunctional [75] or overall voice quality [76].

Our results showed inconsistency between the perceptually assessed variable 'strain' and LTAS measurements: strain/hyperfunction decreased in the participants' voices, but this change was not found in the LTAS parameters. Assessment of the degree of strain/hyperfunction in voice seems to be rather complicated. Although voice experts, according to our clinical experience, recognise it quite easily when working with patients, in many studies, both the reliability between listeners in assessing strain [77] and the correlation of perceptually assessed strain with acoustic parameters [78] have been poor. This concerns especially mildly impaired voices [77] - which our participants' voices represented. It is quite typical for mildly impaired voices that strained/hyperfunctional voice quality is not continuous in speech but appears intermittently at 
the end of sentences. In such a case, a listener might evaluate a specific voice as being more strained than LTAS measures in an acoustic analysis programme would show.

Generally, perceptual evaluation has quite seldom been used in studies assessing the effects of hydration on voice. Based on our literature search, only three studies have used assessment by listening [30-31,79], and in only one of them, listeners could perceive that a change in voice quality had taken place. This was an intervention that comprised systemic dehydration and vocal loading (singing rehearsal without intake of any fluids during two hours before and during the training) [79]. However, no change was detected perceptually in the other two studies. Interestingly, the arrangements of these two last mentioned studies resembled ours more than the first study: one of the studies used nebulization for hydration [31], and the other two were longer-term interventions (five days in the study of Verdolini-Marston et al. [30] and one month in the study of Rocha Santana et al. [31]). It is possible that the combination of warm inhaled steam and the expiratory muscle training contributed to the positive results of our study.

Our intervention seemed to bring out somewhat larger-scale favourable changes in voice variables than earlier hydration studies (see a review in [29]). One explanatory factor may be that our study was carried out during winter when air was constantly dry and therefore generated a similarly demanding environment for all our participants. During the wintertime, outdoor temperature in our area is typically below zero (mean temperature about $14^{\circ} \mathrm{F} /-10^{\circ} \mathrm{C}$ ). This means that the moisture-retaining capacity of air decreases dramatically, followed by a decline of indoor humidity. Cold air contracts capillaries, and this most probably negatively affects the vibration of vocal folds. Additionally, dry winter air has been shown to dehydrate mucosa of the respiratory tract and cause complaints of dryness of throats and noses [80]. It is assumable that cold weather influences laryngeal mucosa in a similar way, especially as the surface of vocal folds rapidly reacts to exposure to dry air $[23,71]$.

Although four out of six of the participants of our study had a lower PPE score after the intervention with the $\mathrm{WellO}_{2}$ device than before it, there was no significant difference between the ratings before and after the intervention. Tanner and colleagues [81], also using the rating of vocal effort using VAS, detected a significant improvement in PPE when nebulized isotonic saline was used in the intervention. This discrepancy may derive from the greater number of participants 
in their study or the differences between saline water and warm steam with no saline which we used. However, our laboratory study with 12 participants with no voice problems showed that, according to most participants, ease of phonation (also reported using a VAS) increased after a short, 15 respiratory cycle training with the $\mathrm{WellO}_{2}$ device [82].

\subsection{The effects of expiratory muscle strength training}

According to our results, the participants reported their breathing to be significantly less effortful after the intervention than before it. Although very few interventions have used the rated respiratory effort, they have revealed both similar [83] and contradictory [84] results. Most of the 12 vocally healthy participants in our earlier study also reported increased ease of respiration after a short $\mathrm{WellO}_{2}$ training [82]. Abdominal breathing activates the vagus nerve [85], the fibres of which have close connections with the trachea and affect both smooth muscle activities and phlegm secretion [86]. We assume that, via both routes, the impact on mucus secretion and airway dilation could have led to subjectively reported less effortful breathing in our study. Namely, secretion of phlegm was one of the complaints many of our participants expressed prior to the intervention, and the self-reported severity of this symptom was somewhat decreased after the $\mathrm{WellO}_{2}$ training.

Furthermore, in the present study, no significant changes were found in the objective pulmonary function tests. Earlier studies also did not find changes in respiratory tests after training [38-39]. It is possible that the pulmonary tests we used may not have been sensitive enough to detect alterations. Namely, in several studies, expiratory muscle training had an impact, but on a different variable, such as maximal expiratory pressure ([38-39], see also [68]). Another reason for our non-significant results may have been caused by the low number of our participants.

Maximum expiratory effort is used in expiratory muscle training with pressure threshold devices [68] because vigorous exhalations are needed to open the pressure relief valve housed in such devices [87-88]. However, the duration of the maximum effort is about one second [87], and it is not considered to require a significant physical and respiratory effort even when the respiratory tasks are repeated [68]. Maximum effort is usually not used in $\mathrm{WellO}_{2}$ exercises. Instead, training is based on long, 10- to 15 -second inhalations and exhalations. To obtain clearer changes in 
respiratory muscle strength, a longer training period is therefore probably needed than the 10minute daily training over a four-week period which we used.

Self-reports of some participants revealed that breathing using the $\mathrm{WellO}_{2}$ device, per se, may have positive effects. One participant pointed out that the deep breathing needed while using the $\mathrm{WellO}_{2}$ calmed her down and enhanced falling asleep in the evenings. This indicates a close connection between respiration and the nervous system: a slow abdominal/diaphragmatic breathing reduces sympathetic activity and enhances vagal activity [85]. Furthermore, deep breathing positively influences physiological and psychological factors by decreasing blood pressure [85], and cortisol [89-90] and stress [90] levels and by improving mood [89-90]. Decreased stress levels may also have had a beneficial effect on our participants' voices because stress is a risk factor for developing a voice disorder. Namely, stress changes the acoustic features of the voice [91-92] and also increases the occurrence of voice symptoms. Cortisol levels are also found to be higher in those individuals who suffer from voice symptoms [93-94]. In further studies, it would be worth obtaining the participants' wider views on the use of the $\mathrm{WellO}_{2}$ device.

\subsection{Generalizability of the results}

As a pilot study, we had only six participants with no control group, so no strong conclusions can be drawn on the basis of the results. The participants were recruited to the present study according to their voice symptoms: their most typical problem was the voice becoming strained, tired or hoarse. These kinds of complaints have been found to be typical of relatively mild functional voice disorders in studies where no vocal pathology has been found [40]. Additionally, in the listening tests, all the subjects were assessed by more than one of the experienced speech therapists as having, at least, mildly disordered voice quality (expressed by the grade component of GRBAS). However, only two of our participants had been examined by a phoniatrician, while others had no formal diagnosis, so we could not confirm the etiology of their voice problems. Despite the missing formal diagnosis, our participants represented a population having asthma [47] and other respiratory and voice symptoms often associated with poor indoor air quality [9596]. Because of these problems, the participants would potentially benefit from breathing 
exercises, phlegm removal and warming and humidification of the vocal tract and the respiratory system.

Although acoustic and perceptual variables showed positive changes in the voice variables in our study, the participants' voice symptoms were not relieved. The intervention time (four weeks) and frequency of exercising (use of $\mathrm{WellO}_{2}$ only once a day for 10 minutes) may have been insufficient for easing voice symptoms. In voice therapy studies showing reduction of symptoms, the length of interventions has been from six weeks to three months [97]. In the respiratory training study where symptoms were alleviated, intervention lasted for five weeks and, consisting of exercises conducted five times a day, was more intensive than that of ours [39]. Further, it is also possible that combining straight vocal exercises with expiratory muscle training is needed to reduce voice symptoms as Wingate and colleagues [41] have shown.

\subsection{Conclusions}

We found favourable effects of humidification of the vocal tract and respiratory training using the WellO $_{2}$ device. Larger-scale studies with a greater number of participants and more severe voice disorders, also including a comparison group, are needed to confirm the results of the present pilot study.

\section{Declaration of interests}

The authors report no conflicts of interests in regard to this work. The $\mathrm{WellO}_{2}$ devices were lent by Hapella Ltd., the manufacturer of the device.

\section{Acknowledgments}

This work was partly supported by the Otological Research Foundation [Korvatautien tutkimussäätiö], Finland, which is kindly acknowledged. We warmly thank speech and language therapists Elina Kankare, Sirpa Pirilä, Kaarina Ruusuvirta and Katja Saarelainen for participating in perceptual assessment of the voice samples. 


\section{Figure captions}

FIGURE 1. WellO 2 device with its cross-section (source: https://wello2.com/product/): 1) double body structure and (2) safety valves of the breathing regulator which protect the user from exposure to hot water, (3) Tritan plastic material free of bisphenol A and estrogenic activity, (4) mouthpiece and breathing regulator, (5) settings for counter pressure, temperature selection and cleaning programme and (6) integrated cleaning basket for sanitation of the device and its accessories. Photo and picture used with written permission from the manufacturer, Hapella Ltd., Kuopio, Finland.

FIGURE 2. Individual AVQI 03.01 total score of the participants $(N=6)$ before the four-week respiratory training period and after it. Horizontal line indicates the AVQI 03.01 cut-off score (1.83), which separates normophonic voices (below the horizontal line) from dysphonic ones (above the line) in adults speaking Finnish. 


\section{REFERENCES}

[1] Schneider SL, Sataloff RT. Voice therapy for the professional voice. Otolaryngol Clin N Am. 2007;40:1133-1149.

[2] Boone D, McFarlane SC, von Berg SL, Zraick RI. The voice and voice therapy, 9th ed. Boston, MA: Pearson; 2014.

[3] Kostyk BE, Rochet AP. Laryngeal airway resistance in teachers with vocal fatigue: A preliminary study. J Voice. 1998;12:287-299.

[4] Iwarsson J, Sundberg J. Breathing behaviors during speech in healthy females and patients with vocal fold nodules. Logoped Phoniatr Vocol. 1999;24:154-169.

[5] Aronson AE, Bless DM. Clinical voice disorders, 4th ed. New York: Thieme; 2009.

[6] Lowell SY, Barkmeier-Kraemer JM, Hoit JD, Story BH. Respiratory and laryngeal function during spontaneous speaking in teachers with voice disorders. J Speech Lang Hear Res. 2008;51:333-349.

[7] Sperry EE, Hillman RE, Perkell JS. The use of inductance plethysmography to assess respiratory function in a patient with vocal nodules. J Med. Speech. Lang. Pathol. 1994;2:137-145.

[8] Stemple JC, Nelson R, Klaben B. Clinical voice pathology: Theory and management, 5th ed. San Diego (CA): Plural Publishing; 2014.

[9] Sapienza CM, Stathopoulos ET, Brown WS Jr. (1997). Speech breathing during reading in women with vocal nodules. J Voice. 11:195-201.

[10] Leydon C, Sivasankar M, Falciglia DL, et al. Vocal fold surface hydration: A review. J Voice. 2009;23:658-665.

[11] Smith S, Thyme K. Die Akzentmethode. Flensburg: Spezial-Pädagogisher Verlag; 1980.

[12] Shiromoto, O. Management of non-organic voice disorders: physiological bases of Accent Method for non-organic voice disorders. Int. Congr. Series. 2003;1240:1269-1276.

[13] Laukkanen A-M, Titze IR, Hoffman H, Finnegan E. Effects of a semioccluded vocal tract on laryngeal muscle activity and glottal adduction in a single female subject. Folia Phoniatr. Logopaed. 2008;60:298311.

[14] Titze IR. Voice training and therapy with a semi-occluded vocal tract: Rationale and theoretical underpinnings. J Speech Lang Hear Res. 2006;49:448-459.

[15] Seikel JA, King DW, Drumright DG. Anatomy \& Physiology for Speech, Language, and Hearing, 4th ed., Clifton Park, New York, Delmar Cengage Learning; 2014.

[16] Sataloff RT. Clinical anatomy and physiology of the voice. In: Sataloff RT, ed. Professional voice. The science and art of clinical care, 3rd ed. San Diego (CA): Plural Publishing; 2005. 
[17] Titze IR. Principles of voice production. Salt Lake City (UT): National Center for Voice and Speech, The University of lowa; 2000.

[18] Hunter EJ, Smith ME, Tanner K. Gender differences affecting vocal health of women in vocally demanding careers. Logoped Phoniatr Vocol. 2011;36:128-136.

[19] Titze IR, Hunter EJ. Comparison of vocal vibration-dose measures for potential-damage risk criteria. J Speech Lang Hear Res. 2015;58:1425-1439.

[20] Vintturi J, Alku P, Sala E, et al. Loading-related subjective symptoms during a vocal loading test with special reference to gender and some ergonomic factors. Folia Phoniatr Logop. 2003;55:55-69.

[21] Sivasankar $M$, Fisher K. Oral breathing increases $P_{\text {th }}$ and vocal effort by superficial drying of vocal fold mucosa. J Voice. 2002;16:172-181.

[22] Tanner K, Roy N, Merrill RM, et al. The effects of three nebulized osmotic agents in the dry larynx. J Speech Lang Hear Res. 2007;50:635-646.

[23] Hemler RJ, Wieneke GH, Lebacq J, et al. Laryngeal mucosa elasticity and viscosity in high and low relative air humidity. Eur Arch Oto-Rhino-Laryngol. 2001;258;125-129.

[24] Sivasankar M, Erickson E, Schneider S, et al. Phonatory effects of airway dehydration: Preliminary evidence for impaired compensation to oral breathing in individuals with a history of vocal fatigue. J Speech Lang Hearing Res. 2008;51:1494-1506.

[25] Witt RE, Taylor LN, Regner MF, et al. Effects of surface dehydration on mucosal wave amplitude and frequency in excised canine larynges. Otolaryngol-Head Neck Surg. 2011;144:108-113.

[26] Leydon C, Wroblewski M, Eichorn N, et al. A meta-analysis of outcomes of hydration intervention on phonation threshold pressure. J Voice. 2010;24:637-643.

[27] Roy N, Weinrich B, Gray SD, et al. Voice amplification versus vocal hygiene instruction for teachers with voice disorders: A treatment outcomes study. J Speech Lang Hear Res. 2002;45:625-638.

[28] Sivasankar M, Leydon C. The role of hydration in vocal fold physiology. Curr Opin Otolaryngol Head Neck Surg. 2010;18:171-175.

[29] Alves M, Krüger E, Pillay B, et al. The effect of hydration on voice quality on adults: A systematic review. J Voice. 2019;33:125.e13-125.e28.

[30] Verdolini-Marston K, Sandage M, Titze IR. Effect of hydration treatments on laryngeal nodules and polyps and related voice measures. J Voice. 1994;8:30-47.

[31] Rocha-Santana É., Vaz Masson ML, Araújo TM. The effect of surface hydration on teachers' voice quality: An intervention study. J Voice. 2017;31:383c5-383c11. doi:10.1016/j.jvoice.2016.08.019.

[32] Bless DM. Measurement of vocal function. Otolaryngol Clin North Am. 1991;24:1023-1033.

[33] Iwarsson J, Thomasson M, Sundberg J. Effects of lung volume on the glottal voice source. J Voice. 1998;12:424-433.

[34] Hammarberg B. Voice research and clinical needs. Folia Phoniatr Logop. 2000;52:93-102. 
[35] Milstein C, Watson PJ. The effects of lung volume initiation on speech: A perceptual study. J Voice. 2004;18:38-45.

[36] Schaeffer N, Cavallo SA, Wall M, et al. Speech breathing behavior in normal and moderately to severely dysphonic subjects during connected speech. J Med Speech-Lang Pathol. 2002;10:1-18.

[37] Sapienza CM, Stathopoulos ET. Respiratory and laryngeal measures of children and women with bilateral vocal fold nodules. J Speech Hear Res. 1994;37:1229-1243.

[38] Baker S, Davenport P, Sapienza S. Examination of strength training and detraining effects in expiratory muscles. J Speech Lang Hear Res. 2005;48:1325-1333.

[39] Tsai YC, Huang S, Che WC, et al. The effects of expiratory muscle strength training on voice and associated factors in medical professionals with voice disorders. J Voice. 2016;30:759.e21-759.e27.

[40] Roy N, Weinrich B, Gray SD. et al. Three treatments for teachers with voice disorders: A randomized clinical trial. J Speech Lang Hear Res. 2003;46:670-688.

[41] Wingate JM, Brown WS, Shrivastav R, et al.Treatment outcomes for professional voice users. J Voice. 2007;21:433-449.

[42] Simberg S, Sala E, Laine A, et al. A fast and easy method for voice disorders among teacher students. Logoped Phoniatr Vocol. 2001;26:10-16.

[43] Ohlsson A-C, Andersson EM, Södersten M, et al. Prevalence of voice symptoms and risk factors of teacher students. J Voice. 2012;26:629-634.

[44] Roy N, Merrill RM, Gray SD, et al. Voice disorders in the general population: Prevalence, risk factors, and occupational impact. Laryngoscope. 2005;115:1988-1995.

[45] Sala E, Laine A, Simberg S, et al. The prevalence of voice disorders among day care center teachers compared with nurses: A questionnaire and clinical study. J Voice. 2001;15:413-423.

[46] Simberg S, Sala E, Tuomainen J, et al. Voice symptoms and allergy - A pilot study. J Voice. 2009;23:136139.

[47] Spantideas N, Drosou E, Bougea A, et al., Inhaled corticosteroids and voice problems. What is new? J Voice. 2017;31:384.e1-384.e7.

[48] Tyrmi J, Laukkanen A-M. How stressful is "deep bubbling"? J Voice. 2017;31:262.e1-262.e6.

[49] Laukkanen A-M, Ilomäki I, Leppänen K, et al. Acoustic measures and self-reports of vocal fatigue by female teachers. J Voice. 2008;22:283-289.

[50] Rantala L, Paavola L, Körkkö P, et al. Working-day effects on the spectral characteristics of teaching voice. Folia Phoniatr Logop. 1998;50:205-211.

[51] Maryn Y, Corthals P, Van Cauwenberge P, et al. Toward improved ecological validity in the acoustic measurement of overall voice quality: Combining continuous speech and sustained vowels. $J$ Voice.

2010;24:540-555. 
[52] Barsties B, Maryn Y. Test-Retest-Variabilität und interne Konsistenz des Acoustic Voice Quality Index. HNO. 2013;61:399-403.

[53] Maryn Y, De Bodt M, Roy N. The Acoustic Voice Quality Index: Toward improved treatment outcome assessment in voice disorders. J Comm Disord. 2010;43:161-74.

[54] Kankare E, Barsties V, Latoszek B, et al. The Acoustic Voice Quality Index version 02.02 in the Finnish speaking population. Logop Phon Vocol. 2019; in press, published online 5 Feb 2019.

[55] Maryn Y, De Bodt M, Barsties B, et al. The value of the Acoustic Voice Quality Index as a measure of dysphonia severity in subjects speaking different languages. Eur Arch Otorhinolaryngol. 2014;271:16091619.

[56] Maryn Y, Weenink D. Objective dysphonia measures in the program Praat: Smoothed cepstral peak prominence and acoustic voice quality index. J Voice. 2015;29:35-43.

[57] Boersma P, Weenink D. Praat: Doing phonetics by computer [Computer program]. Version 6.0.30 [retrieved from http://www.praat.org].

[58] Hirano M. Clinical examination of voice. Wien: Springer-Verlag; 1981.

[59] Karnell MP, Melton SD, Childes JM, et al. Reliability of clinician-based (GRBAS and CAPE-V) and patientbased (V-RQOL and IPVI) documentation of voice disorders. J Voice. 2007;21:576-590.

[60] Joshi A, Watts CR. Measurement reliability of phonation quotient derived from three aerodynamic instruments. J Voice. 2016;30:773.e13-773.e19.

[61] American Thoracic Society/European Respiratory Society: ATS/ERS statement on respiratory muscle testing. Am J Respirat Critical Care Med. 2002;166:518-524.

[62] Suomen kliinisen fysiologian yhdistys ja Suomen Keuhkolääkäriyhdistys: Spirometria- ja PEF-mittausten suoritus ja tulkinta (Execution and interpretation of spirometry and PEF measurements). Suomen kliinisen fysiologian yhdistyksen ja Suomen Keuhkolääkäriyhdistyksen suositus. ed 13. Moodi. 2015;3b:119-142.

[63] Cohen J. A power primer. Psych Bull. 1992;112:155-159.

[64] Koo TK, Li MY. A guide of selecting and reporting intraclass correlation coefficients for reliability research. J Chiropract Med. 2016;15:155-163.

[65] Chaitow L, Bradley D, Gilbert C. What are breathing pattern disorders? In: Chaitow L, Bradley D, Gilbert C, editors. Recognizing and treating breathing disorders: A multidisciplinary approach, 2nd ed. London: Churchill Livingstone; 2013.

[66] Kainu A, Timonen KL, Toikka J, et al. Reference values of spirometry for Finnish adults. Clin Pysiol Funct Imaging. 2015;36:346-258.

[67] Uloza V, Petrauskas T, Padervinskis E, et al. Validation of the Acoustic Voice Quality Index in the Lithuanian language. J Voice. 2017;31:257.e1-257.e11.

[68] Laciuga H, Rosenbek JC, Davenport PW, et al. Functional outcomes associated with expiratory muscle training: Narrative review. J Rehab Res Devel. 2014;51:535-546. 
[69] Mahalingam S, Boominathan P. Effects of steam inhalation on voice quality-related acoustic measures. Laryngoscope. 2016;126:2305-2309.

[70] Franca MC, Simpson KO. Effects of systemic hydration on vocal acoustics of 18- to 35-year-old females. Comm Disord Quarter. 2012;34:29-37.

[71] Hemler RJ, Wieneke GH, Dejonckere PH. The effect of relative humidity of inhaled air on acoustic parameters of voice in normal subjects. J Voice. 1997;11:295-300.

[72] Yiu EM, Chan RM, Effect of hydration and vocal rest on the vocal fatigue in amateur karaoke singers. J Voice. 2003;17:216-227.

[73] Martin D, Fitch J, Wolfe V, Pathologic voice type and the acoustic prediction of severity. J Speech Lang Hear Res. 1995;38:765-771.

[74] Samlan RA, Story B, Bunton K. Relation of perceived breathiness to laryngeal kinematics and acoustic measures based on computational modeling. J Speech Lang Hear Res. 2013;56:1209-1223.

[75] Kitzing P. LTAS criteria pertinent to the measurement of voice quality. J Phon. 1986;14:477-482.

[76] Leino T. Long-term average spectrum in screening of voice quality in speech: Untrained male university students. J Voice. 2009;23:671-676.

[77] Oates J. Auditory-perceptual evaluation of disordered voice quality. Folia Phoniatr Logop. 2009;61:4956.

[78] Bhuta T, Patrick L, Garnett JD. Perceptual evaluation of voice quality and its correlation with acoustic measurements. J Voice. 2004;18:299-304.

[79] van Wyk L, Cloete M, Hattingh D, et al. The effect of hydration on the voice quality of future professional voice performers. J Voice. 2017;31:111.e29-111.e36.

[80] Reinikainen LM, Jaakkola JJ, Heinonen OP. The effect of air humidification on different symptoms in office workers-An epidemiologic study. Environ Int. 1991;17:243-250.

[81] Tanner K, Fujiki RB, Dromey C, et al. Laryngeal desiccation challenge and nebulized isotonic saline in healthy male singers and non-singers: Effects on acoustics, aerodynamics and self-perceived effort and dryness measures. J Voice. 2015;30:670-776.

[82] Huttunen K, Rantala L, Järvinen K, et al. Effects of respiratory training and humidification of the vocal tract with $\mathrm{WellO}_{2}$ device on normal voiced participants. Preliminary findings. PEVOC 12 - Pan-European Voice Congress. August 30th - September 1st, 2017, Ghent, Belgium. Available on-line at: http://www.pevoc.org/pevoc12/abstracts/P19.pdf.

[83] Suzuki S, Sato M, Obuko T. Expiratory muscle training and sensation of respiratory effort during exercise in normal subjects. Thorax. 1995;50:366-370.

[84] Griffiths LA, McConnell AK. The influence of inspiratory and expiratory muscle training upon rowing performance. Eur J Appl Physiol. 2007;99:457-466. 
[85] Wang SZ, Li S, Xu XY, et al. Effect of slow abdominal breathing combined with biofeedback on blood pressure and heart rate variability in prehypertension. J Alt Compl Med. 2010;16:1039-1045.

[86] Wine JJ. Parasympathetic control of airway submucosal glands: Central reflexes and the airway intrinsic nervous system. Auton Neurosci. 2007;133:35-54.

[87] Laciuga H, Davenport PW, Sapienza C. The acute effects of a single session of respiratory muscle strength training on blood pressure, heart rate, and oxygen saturation in healthy adults. Front Physiol. 2012;3:48.

[88] Ma X, Yue Z-Q, Gong Z-Q, et al. The effect of diaphragmatic breathing on attention, negative affect and stress in healthy adults. Front Psychol. 2017;8:874.

[89] Perciavalle V, Blandini M, Fecarotta M, et al.The role of deep breathing on stress. Neurol Sci. 2017;38:451-458.

[90] Mendoza E, Carballo G. Acoustic analysis of induced vocal stress by mean of cognitive workload tasks. J Voice. 1998;12:263-273.

[91] Van Lierde K, van Heule S, De Le S, et al. Effect of psychological stress on female vocal quality. Folia Phoniatr Logop. 2009;61:105-111.

[92] Rantala LM, Hakala SJ, Holmqvist S, et al. Connections between voice ergonomic risk factors and voice symptoms, voice handicap, and respiratory tract diseases. J Voice. 2012;26:819.e13-20.

[93] Gassull C, Casanova C, Botey, Q, et al. The impact of the reactivity to stress in teachers with voice problems. Folia Phoniatr Logop. 2010:62:35-39.

[94] Holmqvist-Jämsén $S$, Johansson A, Santtila $P$, et al. Investigating the role of salivary cortisol on vocal symptoms. J Speech Lang Hear Res. 2017;60:2781-2791.

[95] Patovirta RL. Teachers' health in moisture-damaged schools - A follow-up study. Doctoral dissertation. Publications of the National Public Health Institute A5/2005. Helsinki: National Public Health Institute, 2005. Available on-line at: http://epublications.uef.fi/pub/urn_isbn_951-740-5065/urn_isbn_951-740-506-5.pdf.

[96] Mendell MJ, Mirer AG, Cheung K, et al. Respiratory and allergic health effects of dampness, mold, and dampness-related agents: A review of the epidemiologic evidence. Environ Health Perspect. 2011;119:748756.

[97] Ziegler A, Gillespie Al, Abbott KV. Behavioral treatment of voice disorders in teachers. Folia Phoniatr Logop. 2010;62(1-2):9-23. 